\title{
On the behavior of CO oxidation on shape-controlled Pt nanoparticles in alkaline medium
}

Manuel J.S. Farias, Francisco J. Vidal-Iglesias, José Solla-Gullón, Enrique Herrero, Juan M. Feliu* $^{*}$

Instituto de Electroquímica, Universidad de Alicante Ap. 99, E-03080, Alicante, Spain.

\begin{abstract}
In this work, the behavior of the $\mathrm{CO}$ electro-oxidation reaction on shape-controlled $\mathrm{Pt}$ nanoparticles in alkaline medium was examined in order to understand the effect of the surface structure on this reaction. A series of experiments using Pt nanoparticles of different surface structures/shapes was used and the results obtained were compared with the previous knowledge gained from stepped platinum single crystal electrodes. Independently of the preferential orientation of the nanoparticles, the $\mathrm{CO}$ oxidation voltammetry exhibits two main peaks: one at ca. $0.56-059 \mathrm{~V}$ and the second one at $0.66-0.67 \mathrm{~V}$, being the intensity of the peaks dependent on the shape of the nanoparticle. These two peaks have been assigned to the oxidation of $\mathrm{CO}$ on the (111) terraces and on the rest of the sites, respectively. The appearance of two differentiated peaks reveals that these (111) terraces and the rest of the sites on the nanoparticle surface behave independently of the presence of the other type of sites, that is, they are not connected. The results are discussed considering the effects of the surface mobility of $\mathrm{CO}$ and of the $\mathrm{OH}$ adsorption properties on the different sites in the oxidation peaks.
\end{abstract}

Keywords: Shape-controlled Pt nanoparticles; CO electro-oxidation reaction; alkaline medium. *Corresponding authors: E-mail address: juan.feliu@ua.es (J. M. Feliu). Phone: +34 965909 301. 


\section{Introduction}

The electrochemical adsorption and oxidation of $\mathrm{CO}$ on platinum surfaces is one of the most studied reactions in Electrochemistry. First of all, the electrocatalysis of the CO oxidation reaction is very important for fuel cell technologies, since CO appears as an impurity in hydrogen steams produced from reformed hydrocarbons and also as a poisoning intermediate in the electro-oxidation reaction pathways of many small organic molecules (such as formic acid, methanol or ethanol). Secondly, but not less important, $\mathrm{CO}$ is a simple molecule that can be used, from a fundamental point of view, as a probe molecule to characterize the interfacial properties, such as potential of zero total charge of the Pt surface electrodes, and the electrocatalytic reactivity of the surface sites [1-3].

On platinum electrodes, $\mathrm{CO}$ electro-oxidation reaction is recognized to proceed according to a Langmuir-Hinshelwood (L-H) mechanism [4] in which the adsorbed source of oxygenated species (activated water, as originally proposed by Gilman) reacts with adsorbed CO to yield $\mathrm{CO}_{2}$. It is well-established that $\mathrm{CO}$ electro-oxidation reaction on platinum is a structuresensitive reaction [5]. Such conclusion comes from different studies using basal planes and stepped Pt electrodes [6]. In addition, it is also well-known that the CO electro-oxidation reaction on platinum electrodes strongly depends on the surface composition and the electrolyte solution (acidic or alkaline) [7-9], as well as the nature of both cations [10] and anions [11]. For $\operatorname{Pt}(\mathrm{s})-[(n-1)(111) \times(110)]$ electrodes in acid medium, potentiostatic $\mathrm{CO}$ stripping experiments at full coverage are characterized by the presence of a single $\mathrm{CO}$ oxidation peak $[2,6,12]$. Generally, this single CO stripping peak shifts towards lower potentials as the step density increases thus evidencing that the CO electro-oxidation is influenced by well-ordered [6] or randomly [13] distributed step/kink sites on the electrode surface. Once assumed that the surface diffusion rate of $\mathrm{CO}$ on (111) terrace domains is higher than the rate of $\mathrm{CO}$ electrooxidation reaction [14], it has been accepted that CO electro-oxidation occurs only in step/kink 
sites as nucleation center of oxygen-containing species required for catalyzing $\mathrm{CO}$ oxidation [6]. Additionally, working with $\operatorname{Pt}(\mathrm{s})-[(n-1)(100) \times(110)]$, Vidal-Iglesias et al. [3] demonstrated, that the behavior of the $\mathrm{CO}$ electro-oxidation also depends on the nature of terrace sites. Thus, whereas for (111) terraces [6], the increase of step density always shifts the CO oxidation peak to lower potentials, for (100) terraces, two different trends can be observed for long $(\mathrm{n} \geq 7)$ and short terraces [3]. For long terraces, the CO stripping potential shifts to higher potential values as the step density increases, in strong contrast with the trend observed with the electrodes containing (111) terraces. For short terraces, the "normal" behavior is again observed, that is, as the step density increases the CO stripping peak shifts towards lower potentials, although splitting into two separate contributions. These observations evidence an especial activity of the (100) terrace sites in comparison to that attributed to the (111) ones. However, in all cases above, the current-transient behavior as well as the voltammograms of $\mathrm{CO}$ oxidation can be well fitted with a mean-field $\mathrm{L}-\mathrm{H}$ model $[6,15]$ which assumes a random distribution of the adsorbed $\mathrm{OH}$ and $\mathrm{CO}$ species. Consequently, according with the mean field approximation [16], an effective homogeneous distribution of the species can be obtained. In this sense, it is important to note that the dissociation of water to form $\mathrm{OH}_{\mathrm{ads}}$ is thermodynamically favorable at defect sites (kink, step and edge sites) in comparison to terrace sites [17].

The voltammetry for $\mathrm{CO}$ oxidation on platinum electrodes in alkaline medium is, at first glance, much more complex than that obtained in acid medium. The CO stripping voltammogram in alkaline medium shows multiple oxidation peaks that can be correlated to the oxidation of $\mathrm{CO}$ on different surface sites, being even possible to assign each peak to the oxidation of $\mathrm{CO}$ with a specific surface site. Assuming that step sites act as center for nucleation of oxygen-containing species to catalyze $\mathrm{CO}$ oxidation, the existence of these multiple peaks has been related to differences in the $\mathrm{CO}$ surface diffusion rate between acidic and alkaline media [18], being the latter much slower that the former and also slower than the reaction rate 
[19]. For stepped and kinked Pt surfaces with (111) terraces and (110) or (100) steps, up to three different $\mathrm{CO}$ oxidation peaks located at $0.58,0.70$ and $0.78 \mathrm{~V}$ ( $v s$ RHE) have been observed and assigned to the $\mathrm{CO}$ electro-oxidation with kink/step sites and (111) terrace sites, respectively, [18]. Recently, a series of experiments have allowed reassigning the site release for a particular oxidation peak [20,21]. For Pt(111) vicinal surfaces, for which electronic effects might modify both stability and structure of adsorbed $\mathrm{CO}$ at the (111) terrace sites, $\mathrm{CO}$ adsorbs firstly at low-coordination sites and its oxidation occurs preferentially at (111) terrace sites close to the lower side of the low-coordination (kink/step) sites [20]. Thus, CO molecules adsorbed on top of the step sites were the last ones to be oxidized. Interestingly, in the case of $\mathrm{CO}$ electro-oxidation reaction at $\mathrm{Pt}(100)$ and its vicinal surfaces in alkaline medium [22], some relevant peculiarities are observed for such electrodes, as previously observed in acidic medium.

Taking into account the remarkable structure sensitivity of $\mathrm{CO}$ electro-oxidation reaction on Pt electrodes, it would be expected a similar trend with shape-controlled Pt nanoparticles for which their surface structure and particularly their preferential (100) or (111) terrace contributions are similar to the single crystal surfaces [23]. In acidic medium, CO oxidation reaction on these shape-controlled Pt nanoparticles showed a clear $\mathrm{CO}$ oxidation peak multiplicity that was attributed to CO oxidation from ordered (100) and (111) domains as well as from disordered surface domains [23-26]. As found on Pt single crystal electrodes, the sharp $\mathrm{CO}$ oxidation peak at high potentials was assigned to the $\mathrm{CO}$ oxidation on two-dimensional (100) terraces because this contribution well correlates with the amount and quality of the (100) domains present at the surface of the nanoparticles. The correlation between $\mathrm{CO}$ peak multiplicity and particle surface structure was reproduced and confirmed in other contributions by different groups $[24,26]$. However, CO peak multiplicity is a very controversial topic and, on non-preferentially oriented Pt nanoparticles, several explanations have been proposed to 
explain the origin of the peak multiplicity. As recently summarized by Maillard et al. [27] and Koper [28], contradictory results have been reported for $\mathrm{CO}$ electro-oxidation on $\mathrm{Pt}$ nanoparticles. However, it is important to point out that the understanding of the process is not a simple question because several aspects such as particle agglomeration, reduced CO diffusion on small particles $(<4 \mathrm{~nm})$, surface structure, as well as contribution of defect sites affect the resulting reactivity.

In alkaline solution, $\mathrm{CO}$ electro-oxidation reaction on shape-controlled $\mathrm{Pt}$ nanoparticles has been very recently reported for the first time [29]. Results suggest that the behavior of the preferentially (111) and (100) oriented nanoparticles strongly deviates from that expected from the Pt single-crystal electrodes. Consequently, in this paper we report a comprehensive discussion and detailed insights into the CO stripping oxidation in alkaline medium on different shape controlled Pt nanoparticles.

\section{Experimental section}

\subsection{Synthesis and cleaning of the Pt nanoparticles}

The experimental details for the preparation and surface cleaning of the different nanoparticles used in this contribution have been given in some previous contributions. Very briefly, Pt nanoparticles with preferential spherical shape (termed $\mathrm{PtNP}_{\text {sphe }}$ ) were synthesized by reducing $\mathrm{H}_{2} \mathrm{PtCl}_{6}$ with sodium borohydride using a water-in-oil (w/o) microemulsion [3033]. The shape-controlled Pt nanoparticles [with preferential cubic shape (termed $\mathrm{PtNP}_{\text {cubic }}$ ), with preferential octahedral tetrahedral shape (termed $\mathrm{PtNP}_{\text {tetra }}$ ) and with preferential octahedral and tetrahedral truncated shape (termed $\left.\mathrm{PtNP}_{\text {trunc }}\right)$ ] were synthesized by using a colloidal method with sodium polyacrylate $\left(\mathrm{Mw}=2100 \mathrm{~g} \mathrm{~mol}^{-1}\right)$ as capping agent, $\mathrm{K}_{2} \mathrm{PtCl}_{4} \mathrm{or}_{2} \mathrm{H}_{2} \mathrm{PtCl}_{6}$ as metallic precursor, and $\mathrm{H}_{2}$ as reducing agent $[32,34,35]$. The synthesis of the smallest $\mathrm{Pt}$ nanoparticles supported on carbon has been also detailed in previous contribution $[30,36]$ and 
it is based on the reduction of $\mathrm{H}_{2} \mathrm{PtCl}_{6}$ with ice cold sodium borohydride in presence of sodium citrate. After the reduction step, carbon Vulcan XC-72R was added and stirred to properly disperse the nanoparticles on the carbon support.

The different strategies to clean the Pt nanoparticles have been also found in previous contributions and strongly depend on the synthetic route employed [30, 31, 34]. Thus, whereas the nanoparticles obtained in microemulsion were cleaned with successive additions of acetone, acetone-water mixtures and water to finally achieve a water suspension containing the nanoparticles, the cleaning of the preferentially shaped nanoparticles was performed by using a strong basic aqueous solution $(\mathrm{NaOH})$ followed by several water washes to finally achieve a water suspension containing the nanoparticles. Finally, the cleaning of the nanoparticles prepared in presence of citrate was also performed using a strong basic aqueous solution $(\mathrm{NaOH})$. However, in case of the carbon supported sample, it was also filtered, rinsed at least 3-4 times with ultra-pure water, and finally dried at $70-80^{\circ} \mathrm{C}$.

\subsection{Characterization of Pt nanoparticles}

A detailed characterization of the different nanoparticles used in this paper can be found in previous contributions $[29,31,37]$. The particle size and shape of the different $\mathrm{Pt}$ nanoparticles have been characterized by Transmission Electron Microscopy (TEM) using a JEOL, JEM 2010 microscope working at $200 \mathrm{kV}$. The sample was obtained by placing a drop of the dispersed solution onto a Formvar-covered copper grid and evaporating it in air at room temperature. For each sample, usually more than 200 particles from different parts of the grid were used to estimate the mean diameter and size distribution of the nanoparticles. Very briefly, the particle size of the $\mathrm{PtNP}_{\text {sphe }}, \mathrm{PtNP}_{\text {cubic }}, \mathrm{PtNP}_{\text {tetra }}$ and $\mathrm{PtNP}_{\text {trunc }}$ nanoparticles is about 4-5 nm for the spherical particles and about 8-10 $\mathrm{nm}$ for the shaped nanoparticles. In the case of the 
particles prepared in presence of citrate for both supported and unsupported, the particle size is about 2-3 nm.

\subsection{Electrochemical characterization}

The electrochemical characterization of the samples was performed in a three-electrode electrochemical cell. The counter electrode was a large gold wire. Potentials were measured against a reversible hydrogen electrode (RHE) connected to the cell through a Luggin capillary. For the Pt nanoparticles, a droplet ranging from 1 to $3 \mu \mathrm{L}$ of the solution containing the nanoparticles was deposited on a hemispherical polycrystalline gold electrode or on glassy carbon substrate and dried under Ar atmosphere. Before each experiment, the gold collector or glassy carbon was mechanically polished with alumina and rinsed with ultra-pure water to eliminate the nanoparticles from previous experiments. In order to perform the adsorbed $\mathrm{CO}$ oxidation experiments, $\mathrm{CO}(\mathrm{g})$ was admitted into the atmosphere cell at $0.100 \mathrm{~V}$ until the complete or partial blockage of the surface was reached, which was monitored by charge displacements during $\mathrm{CO}$ adsorption. After that, $\mathrm{CO}$ was removed from the solution by bubbling Ar for at least $18 \mathrm{~min}$. CO stripping voltammograms were registered at 0.02 or 0.05 $\mathrm{V} \mathrm{s}^{-1}$ in order to partial or totally oxidize the $\mathrm{CO}$ adlayer on the surface. The supporting electrolytes, $0.5 \mathrm{M} \mathrm{H}_{2} \mathrm{SO}_{4}$ (Merck KGaA 96\%) and 0.1 M NaOH (Merck KGaA, $99.99 \%$ ) were prepared in ultrapure water (Millipore Milli-Q $18.2 \mathrm{M} \Omega \mathrm{cm}$ ). $\mathrm{In}_{2} \mathrm{H}_{2} \mathrm{SO}_{4}$ solution, the active surface area of the different Pt nanoparticles was determined by the charge involved in the socalled hydrogen UPD (Under Potential Deposition) region until $0.63 \mathrm{~V}$ assuming $230 \mu \mathrm{C} \mathrm{cm}-2$ for the total charge after the subtraction of the double layer charging contribution as previously discussed [23]. In alkaline solution, the total charge measured between 0.06 and $0.90 \mathrm{~V}$ (without any double layer correction) was normalized to $390 \mu \mathrm{C} \mathrm{cm}^{-2}$ as recently proposed by VidalIglesias et al. [29]. 


\section{Results and discussions}

\subsection{Voltammetric characterization of surface structure of the nanoparticles}

Figure 1 shows the voltammetric profiles of the $\mathrm{PtNP}_{\text {sphe }}, \mathrm{PtNP}_{\text {cubic }}, \mathrm{PtNP}_{\text {tetra }}$ and $\mathrm{PtNP}_{\text {trunc }}$ nanoparticles in acidic (black line) and alkaline (red line) solutions. In all cases, the sharpness, good definition and the symmetry of the adsorption/desorption states in all samples are clear evidences of the effective surface cleanliness. As described in previous contribution [31], each particular voltammetric profile reflects the characteristic/specific surface structure of each sample. Consequently, in terms of surface structure, the $\mathrm{PtNP}_{\text {sphe, }}, \mathrm{PtNP}_{\text {cubic }}, \mathrm{PtNP}_{\text {tetra }}$ and PtNP $_{\text {trunc }}$ samples can be assigned to polyoriented, (100), (111), and (111)-(100) preferentially oriented Pt nanoparticles, respectively [31].

In addition, it is worth noting that although the surface structure of the nanoparticles is always much more complex than that of the platinum single crystal electodes, the voltammetric features obtained with the different Pt nanoparticles perfectly correlate with what would be expected from the dominant shape/symmetry domain of the nanoparticles and the knowledge of the voltammetric responses of the Pt single crystal electrodes.

\subsection{CO stripping voltammetry at shape-controlled Pt nanoparticles}

Figure 2 shows the $\mathrm{CO}$ stripping voltammetry with different shape-controlled $\mathrm{Pt}$ nanoparticles in alkaline medium. The general profiles are very similar to those previously reported [29]. However, some important features should be pointed out and discussed in more detail. For all CO stripping voltammetries, there are two well defined peaks (named by I and II) at $\sim 0.57 \mathrm{~V}$ and $\sim 0.67 \mathrm{~V}$. At potentials lower than the peak II there is a small (but very important) contribution started $\sim 0.33 \mathrm{~V}$ and usually named pre-wave. For CO electro-oxidation reaction at $\mathrm{Pt}(111)$ and its vicinal electrodes in alkaline medium, the contribution at $\sim 0.5-0.7 \mathrm{~V}$ 
has been clearly assigned to the CO oxidation at (111) terrace sites when step/kink sites are present on those terraces $[20]$. For these surfaces, the contribution coming from CO electrooxidation with (110) or (100) step sites appears only at $\sim 0.8 \mathrm{~V}[20]$. However, the feature at around $0.8 \mathrm{~V}$ is never observed at Pt nanoparticles independently of their shape. In this regard, and taking into account that some of the samples (for examples as $\mathrm{PtNP}_{\text {tetra }}$ and $\mathrm{PtNP}_{\text {trunc }}$ ) have an important fraction of (111) domains as well as others have a high fraction of surface steps/defects, the absence of contributions at around $0.8 \mathrm{~V}$ clearly indicates that the behavior of the nanoparticles cannot be directly extrapolated from the reactivity previously reported with the single crystal surfaces and that therefore an additional factor is affecting/altering their reactivity towards $\mathrm{CO}$ electro-oxidation. In fact, the results obtained in Fig. 2 suggest that the $\mathrm{CO}$ electro-oxidation reaction on Pt nanoparticles in alkaline solution seems to be quite insensitive to the surface structure of the nanoparticles, in contrast with what would be expected from previous Pt single crystal studies [18-20]. Moreover, as previously reported by Chen et $a l$. [38] the CO stripping voltammetric profile obtained with a Pt thin film supported on Glassy Carbon (GC) in $0.1 \mathrm{M} \mathrm{NaOH}$ is almost identical to those obtained with the different $\mathrm{Pt}$ nanoparticles in the present work (Fig. 2). Very recently, Cantane and Lima [39] also showed a similar voltammetric profile for electrodeposited Pt electrodes (about $10 \mathrm{~nm}$ thick) in $0.1 \mathrm{M}$ $\mathrm{NaOH}+0.9 \mathrm{M} \mathrm{NaClO}_{4}$

To better understand this issue, Fig. 3 shows a comparison between the CO stripping voltammetric responses (Fig. 3B) of a Pt polycrystalline electrode (Pt bead) and that obtained with spherical Pt nanoparticles with a particle size around 2-3 nm, that is, nanoparticles with a high fraction of step/edge sites and consequently a very low contribution from surface domains. Fig. 3A is referred as the blank voltammetry of the samples. Very interestingly, and similarly to the results reported in Fig. 2, the $\mathrm{CO}$ electro-oxidation behavior is very similar, independently of the electrode structure. The very small feature (highlighted in inset of Fig. 3B) for the Pt 
bead can be observed at $0.79 \mathrm{~V}$, which can be related to the presence of a very small fraction of large (111) terrace domains on these surfaces. As aforementioned, step/kink sites on those domains give an oxidation peak at ca. $0.79 \mathrm{~V}$. Again, this feature is absent in the case of $\mathrm{Pt}$ nanoparticles. The $\mathrm{CO}$ voltammetric profile of the polycrystalline Pt electrode is essentially similar to that previously reported in [9] with similar electrodes and in similar $\mathrm{pH}$ conditions.

This clear absence of $\mathrm{CO}$ oxidation contributions at potentials around $0.8 \mathrm{~V}$ and independently of the structure of nanoparticles should suggest that the CO surface mobility from any site toward more active sites on the Pt nanoparticles surfaces should be expected to be much higher than that considered on Pt single crystals. However, the fact that (111) terrace sites become very reactive as compared to step sites in stepped Pt electrodes evidences that this high reactivity is not related with a high CO mobility on the surface [20]. Thus, the fact that at Pt nanoparticles all the $\mathrm{CO}$ molecules into the adlayer were oxidized at potentials lower than $0.8 \mathrm{~V}$ could not have any relationship with a higher $\mathrm{CO}$ diffusion coefficient on the surface of the nanoparticles.

Insights about these issues can be drawn by experiments at different scan rates. $\mathrm{CO}$ stripping profiles at different scan rates on the $\mathrm{PtNP}_{\text {cubic }}$ (Fig. 4) and $\mathrm{PtNP}_{\text {trunc }}$ (Fig. 5) samples show again clear discrepancies with the behavior observed for single crystal electrodes [20]. In all cases, the only contributions are those appearing at low scan rates. In fact, the only effect of the scan rate is the shift in the peaks' potential. Unlike the behavior observed for the stepped surfaces [18], there is not a significant change in the charge of each peak with the scan rate. Additionally, the apparent Tafel slopes $(\mathrm{d} E / \mathrm{d} \log v)$ for the different peak processes are different from those measured on the stepped single crystal electrodes. For those electrodes, the Tafel values are ca. $90 \mathrm{mV}$ for the peak related to the oxidation of $\mathrm{CO}$ on the (111) terraces with steps and ca. 30-40 mV for that on the steps [20]. On the other hand, the apparent Tafel slopes for $\mathrm{CO}$ electro-oxidation in Fig. 4 and 5 are between $75-90 \mathrm{mV}$ for both peaks. The difference 
clearly indicates that the mechanism for the $\mathrm{CO}$ oxidation on the stepped surfaces and on the nanoparticles is different. These differences are probably related to a different $\mathrm{OH}$ adsorption isotherm on those surfaces [40].

Another point that confirms the differences between nanoparticles and single crystal electrodes in alkaline medium comes from a more detailed analysis of the response of the $\mathrm{PtNP}_{\text {cubic }}$ to the $\mathrm{CO}$ electro-oxidation reaction. In stepped surfaces with (100) terraces and (111) steps, a single $\mathrm{CO}$ oxidation peak is observed whereas multiple peaks are obtained for platinum surfaces with (111) terraces and (110) and/or (100) steps [3]. The behavior of the CO oxidation reaction on $\mathrm{Pt}(100)$ vicinal electrodes was explained in terms of a faster surface mobility of CO on the (100) surface domains than in the (111) ones [3]. This single oxidation peak shifts towards lower potential values when the (111) step density increases. It appears between 0.62$0.67 \mathrm{~V}$ for terrace widths between 6 to 20 atomic rows, respectively. However, in $\mathrm{PtNP}_{\text {cubic }}$ nanoparticles multiple $\mathrm{CO}$ oxidation peaks are observed, in clear contradiction with the single crystal results. In addition, these unexpected contributions appear at more positive potentials than those measured with the stepped single crystal electrodes. This would suggest that, in this particular case, the CO mobility on the (100) domains on the surface of the nanoparticles is now lower than that observed on single crystals having (100) terraces. The different trends observed with the nanoparticles containing preferential (111) and (100) orientations, made us consider/evaluate other options as it would be described below.

\subsection{Site CO occupancy on shape-controlled Pt nanoparticles and its effect on the voltammetric profile}

As previously stated in the introduction section, there is a clear relationship between the oxidation peak potential and the adsorption site on stepped Pt surfaces having (111) terraces and monoatomic (110) or (100) steps $[20,21]$. Based on experimental results, the slow CO 
oxidation releases first the (111) terrace sites and, only when all the terrace sites are free, $\mathrm{CO}$ oxidation occurs on the (110) and (100) step sites. In addition, the CO adsorption/filling on these surfaces occurrs preferentially with the following sequence: first (100) step/kink sites, then (110) step sites and finally (111) terrace domains. To evaluate whether the CO adsorption/filling on the surface sites of nanoparticles has a similar behavior to that observed with stepped Pt electrodes, we have performed additional experiments, as described in [20]. In these experiments, a low CO gas flow was introduced in the electrochemical cell atmosphere for a given time. Since the CO concentration in solution is very low, the increase in the $\mathrm{CO}$ coverage during the required time to record the stripping peak is negligible, so that the blockage of the nanoparticle surface can be monitored by inspecting the hydrogen adsorption region. In this way, the different sites blocked by $\mathrm{CO}$ were monitored vs. the dosing time. Figure 6 shows the results obtained for the $\mathrm{PtNP}_{\text {cubic }}$ and $\mathrm{PtNP}_{\text {tetra }}$ and compared with a kinked $\mathrm{Pt}$ electrode $\operatorname{Pt}(17,13,11) \equiv \operatorname{Pt}(s)-[6(111) \times 2(110) \times 1(100)]$. The results clearly indicate that on the shaped $\mathrm{Pt}$ nanoparticles the $\mathrm{CO}$ adsorption/filling does not occur preferentially, in contrast with what is observed with the single crystal (Fig. 6C).

These results indicate that there is no significant difference in $\mathrm{CO}$ adsorption energy for different structural sites at the surface of the nanoparticles, that is, the adsorption process occurs randomly on the surface. In addition, the $\mathrm{CO}$ electro-oxidation reaction profile corresponding to each particular $\mathrm{CO}$ sub-monolayer coverage shows a continuous growth of all $\mathrm{CO}$ oxidation contributions which also indicates the absence of a preferential surface site for $\mathrm{CO}$ oxidation (Fig. 6A-B), i.e., the growth of $\mathrm{CO}$ electro-oxidation peaks at 0.57 and 0.67 occurs simultaneously.

To complete the comparison between the Pt nanoparticles and stepped Pt electrodes, a new set of experiments was performed, in which $\mathrm{CO}$ was partially stripped off from the electrode surfaces (Fig. 7). The electrode was first fully blocked by $\mathrm{CO}$ and then the dissolved 
$\mathrm{CO}$ was removed by Ar purging at controlled potential $(0.1 \mathrm{~V})$. For the cubic and octahedral $\mathrm{Pt}$ nanoparticles (Fig. 7A and B), the CO adlayer was partially removed by recording a stripping voltammogram until the complete oxidation of $\mathrm{CO}$ under peak II. At this point, the potential was stepped to $0.1 \mathrm{~V}$ and the remaining $\mathrm{CO}$ was stripped off from the electrode surface in a single sweep by increasing the electrode potential up to $0.86 \mathrm{~V}$ (red line). The stripping voltammogram for partial $\mathrm{CO}$ coverages can be compared with that measured for the full $\mathrm{CO}$ coverage (black line). The partial stripping experiment is also shown for the kinked $\operatorname{Pt}(17,13,11)$ electrode in Fig. 7C. From these results, some important features can be pointed out. The first one is that related to the so-called hydrogen adsorption region. As can be observed in Fig. 7A and $\mathrm{B}$, after the removal of the contribution II, the hydrogen region shows that the peaks at 0.27 and $0.42 \mathrm{~V}$ still remain blocked by the remaining adsorbed $\mathrm{CO}$ [29]. The peak at $0.27 \mathrm{~V}$ is related to (110) sites whereas the peak at $0.42 \mathrm{~V}$ corresponds to processes related to (100) step sites and (100) small domains [29]. The observed hydrogen adsorption after partial stripping can then be related to the adsorption on (111) terraces. In alkaline medium, the only contributions that appear below $0.2 \mathrm{~V}$ are those associated with (111) ordered domains. Thus, this evidence suggests that the process II is preferentially due to the $\mathrm{CO}$ oxidation on the (111) terrace sites. A similar finding is obtained with the kinked surface where the $\mathrm{CO}$ partial stripping was limited to the process III. In agreement with previous observations [20], the hydrogen region associated with the (110) and (100) step sites remained blocked while the (111) terrace sites are now fully accessible to hydrogen adsorption. Consequently, this contribution III was also assigned to the CO oxidation liberating (111) terrace sites.

The most relevant point is that related to the $\mathrm{CO}$ stripping of the remaining $\mathrm{CO}$. As previously stated, it seems that the remaining $\mathrm{CO}$ is mainly adsorbed on the sites with (110) and (100) orientations. Thus, peak I can be related to the oxidation of $\mathrm{CO}$ on these sites. It should be highlighted that the intensity of the peak is related to the amount of (100) domain 
sites on the sample; the peak intensity is the highest for the $\mathrm{PtNP}_{\text {cubic }}$ and $\mathrm{PtNP}_{\text {trunc. In addition, }}$ after partial removal of $\mathrm{CO}$ at (111) terrace sites, it is observed an important shift of potential toward lower values for process I. Such potential shift for CO electro-oxidation reaction with (110) or (100) step sites is not observed for stepped Pt electrodes with (111) terraces [20], in agreement with the response showed in Fig. $7 \mathrm{C}$ for the kinked $\mathrm{Pt}$ electrode. For the $\mathrm{CO}$ oxidation reaction, the peak potential depends not only on the catalytic activity of the surface, but also on the initial amount of defects on the adlayer to initiate the oxidation process [40-42]. Thus, the peak shift can be related to an initial disordering of the remaining adlayer that facilitates the ignition of the oxidation process.

The fact that the contributions of the (100) and (110) sites appear at the same potential, clearly suggests that these types of sites are interconnected. These results are in agreement with the observed behavior for the stepped (100) terraces [22], for which a single peak is observed. Two different possibilities appear to explain this interconnection. The first one is that diffusion between (100) terraces and low coordination (100) and (110) sites is possible, so that CO moves rapidly towards the most reactive sites. This situation will contrast with the behavior observed for the (111) terraces, on which CO diffusion is very slow [19]. The other possibility is that the oxidation on one of those sites will ignite the oxidation of the rest, through a nucleation and growth mechanism. The major difference between (111) terrace sites and the rest of the sites is the onset for $\mathrm{OH}$ adsorption. When $\mathrm{CO}$ is oxidized on a (111) terrace site at $0.56-0.57 \mathrm{~V}, \mathrm{OH}$ adsorption will not occur on this new released site, since the onset is ca. $0.7 \mathrm{~V}$. Thus, these sites cannot be used to oxidize the neighboring $\mathrm{CO}$ molecules. However, for the remaining sites, $\mathrm{OH}$ can readily adsorb on those sites when $\mathrm{CO}$ is oxidized above $0.6 \mathrm{~V}$, and ignite the oxidation on neighboring sites. This way, once CO has been oxidized on one of those sites, the oxidation wave propagates over the surface resulting in a single peak. This single peak is compatible with a low diffusion on the surface. 


\subsection{Effect of nanoparticle agglomeration}

From previous contributions [28, 43, 44], it is well established that, in acid medium, the nanoparticle agglomeration plays a significant role on the $\mathrm{CO}$ stripping potential. It has been reported that the $\mathrm{CO}$ electro-oxidation reaction at Pt nanoparticles unsupported or supported on carbon depends on the platinum loading (from 10 to 50\%). In this way, a single peak at ca. 0.8 $\mathrm{V}$ is observed for the samples with low loading whereas, a second peak at lower potentials continuously grows as a function of the agglomeration. This peak at low potentials is well resolved for the 50\% loading $\mathrm{Pt} / \mathrm{C}$ sample as well as with the unsupported nanoparticles. This low-potential $\mathrm{CO}$ peak was attributed to a reaction between adsorbed $\mathrm{OH}$ and $\mathrm{CO}$ species on different but close nanoparticles, that is, an inter-particle $\mathrm{CO}$ oxidation process [43]. To elucidate the possible effect of the agglomeration of the nanoparticles, we have also compared the voltammetric profiles for $\mathrm{CO}$ electro-oxidation between those supported on carbon with a metal loading of $10 \%$ wt in which the Pt nanoparticles are mainly isolated and unsupported $\mathrm{Pt}$ nanoparticles where the agglomeration is maximum (figure 8). The results indicate that whereas in acidic solution the CO stripping on isolated Pt nanoparticles shifts to higher potential (Fig. 8D), in alkaline solution the position of the $\mathrm{CO}$ oxidation peak remains practically unaltered (Fig. 8C), suggesting that the level of agglomeration of the nanoparticles does not play an important role in alkaline solutions and consequently that an inter-particle process should not be considered to affect the resulting activity as observed in acidic solution [43].

\section{Conclusions and additional remarks.}

A series of experiments have been carried out in order to reach a better understanding on the electrochemical behavior of well-defined Pt nanoparticles towards $\mathrm{CO}$ electro-oxidation in alkaline media. The obtained results were compared with those previously reported using 
stepped and kinked $\mathrm{Pt}$ electrodes. Independently of the preferential orientation of the nanoparticles, the $\mathrm{CO}$ oxidation voltammetry exhibits two main peaks: one at ca. $0.56-059 \mathrm{~V}$ and the second one at $0.66-0.67 \mathrm{~V}$. Using the knowledge gained with the stepped surfaces, these two peaks have been assigned to the oxidation of $\mathrm{CO}$ on the (111) terraces and on the rest of the sites, respectively. This two separate contributions clearly suggest that $\mathrm{CO}$ on the (111) terrace siets and $\mathrm{CO}$ on the rest of the sites are not connected, that is, diffusion between (111) terrace sites and the rest is not possible. The other sites, namely (100) terrace sites and (110) and (100) low coordination sites are interconnected for CO oxidation because only one peak is observed for the oxidation of $\mathrm{CO}$ on those different sites. Additionally, there is no preferential adsorption of $\mathrm{CO}$ on the different surface sites, that is, $\mathrm{CO}$ adsorption occurs randomly on the surface.

Acknowledgements: Farias, M.J.S. would like to thanks CNPq, Brazil, for financial support for his postdoctoral stay at Universidad de Alicante. This work has been financially supported by the MICINN (Spain) (project CTQ2010-16271) and Generalitat Valenciana (project PROMETEO/2009/045, FEDER).

\section{References}

[1] R. Gómez, V. Climent, J.M. Feliu, M.J. Weaver, J. Phys. Chem. B, 104 (2000) 597.

[2] N.P. Lebedeva, M.T.M. Koper, J.M. Feliu, R.A. van Santen, J. Phys. Chem. B, 106 (2002) 12938.

[3] F.J. Vidal-Iglesias, J. Solla-Gullón, J.M. Campina, E. Herrero, A. Aldaz, J.M. Feliu, Electrochim. Acta, 54 (2009) 4459.

[4] S. Gilman, J. Phys. Chem. B, 68 (1964) 70.

[5] J.M. Leger, B. Beden, C. Lamy, S. Bilmes, J. Electroanal. Chem., 170 (1984) 305.

[6] N.P. Lebedeva, A. Rodes, J.M. Feliu, M.T.M. Koper, R.A. van Santen, J. Phys. Chem. B, 106 (2002) 9863.

[7] J.S. Spendelow, J.D. Goodpaster, P.J.A. Kenis, A. Wieckowski, J. Phys. Chem. B, 110 (2006) 9545.

[8] A. Couto, A. Rincón, M.C. Pérez, C. Gutiérrez, Electrochim. Acta, 46 (2001) 1285. 
[9] R. Gisbert, G. García, M.T.M. Koper, Electrochim. Acta, 56 (2011) 2443.

[10] C. Stoffelsma, P. Rodriguez, G. Garcia, N. Garcia-Araez, D. Strmcnik, N.M. Marković, M.T.M. Koper, J. Am. Chem. Soc., 132 (2010) 16127.

[11] N.M. Marković, C.A. Lucas, A. Rodes, V. Stamenkoví, P.N. Ross, Surf. Sci., 499 (2002) L149.

[12] N.P. Lebedeva, M.T.M. Koper, J.M. Feliu, R.A. van Santen, J. Electroanal. Chem., 524 (2002) 242.

[13] A. López-Cudero, A. Cuesta, C. Gutiérrez, J. Electroanal. Chem., 579 (2005) 1.

[14] A.V. Petukhov, W. Akemann, K.A. Friedrich, U. Stimming, Surf. Sci., 402 (1998) 182.

[15] M.T.M. Koper, N.P. Lebedeva, C.G.M. Hermse, Faraday Discuss., 121 (2002) 301.

[16] M.T.M. Koper, A.P.J. Jansen, R.A. van Santen, J.J. Lukkien, P.A.J. Hilbers, J. Chem. Phys., 109 (1998) 6051.

[17] M. van der Niet, A. den Dunnen, L.B.F. Juurlink, M.T.M. Koper, J. Chem. Phys., 132 (2010).

[18] G. Garcia, M.T.M. Koper, Phys. Chem. Chem. Phys., 10 (2008) 3802.

[19] E. Herrero, Q.-S. Chen, J. Hernandez, S.-G. Sun, J.M. Feliu, Phys. Chem. Chem. Phys., 13 (2011) 16762.

[20] M.J.S. Farias, E. Herrero, J.M. Feliu, J. Phys. Chem. c, 117 (2013) 2903.

[21] G. Garcia, M.T.M. Koper, J. Am. Chem. Soc., 131 (2009) 5384.

[22] R.M. Arán-Ais, M.C. Figueiredo, F.J. Vidal-Iglesias, V. Climent, E. Herrero, J.M. Feliu, Electrochim. Acta, 58 (2011) 184.

[23] Q.S. Chen, F.J. Vidal-Iglesias, J. Solla-Gullón, S.G. Sun, J.M. Feliu, Chem. Sci., 3 (2012) 136.

[24] S. Kinge, C. Urgeghe, A. De Battisti, H. Bönnemann, Appl. Organomet. Chem., 22 (2008) 49.

[25] S. Brimaud, S. Pronier, C. Coutanceau, J.M. Léger, Electrochem. Commun., 10 (2008) 1703.

[26] M. Nakamura, Y. Hanioka, W. Ouchida, M. Yamada, N. Hoshi, ChemPhysChem, 10 (2009) 2719.

[27] F. Maillard, E.R. Savinova, U. Stimming, J. Electroanal. Chem., 599 (2007) 221.

[28] M.T.M. Koper, Nanoscale, 3 (2011) 2054.

[29] F.J. Vidal-Iglesias, R.M. Aran-Ais, J. Solla-Gullon, E. Herrero, J.M. Feliu, ACS Catal., 2 (2012) 901.

[30] J. Solla-Gullón, V. Montiel, A. Aldaz, J. Clavilier, J. Electrochem. Soc., 150 (2003) E104.

[31] J. Solla-Gullón, P. Rodríguez, E. Herrero, A. Aldaz, J.M. Feliu, Phys. Chem. Chem. Phys., 10 (2008) 1359.

[32] J. Solla-Gullón, F.J. Vidal-Iglesias, A. López-Cudero, E. Garnier, J.M. Feliu, A. Aldaz, Phys. Chem. Chem. Phys., 10 (2008) 3689.

[33] F.J. Vidal-Iglesias, J. Solla-Gullon, J.M. Perez, A. Aldaz, Electrochem. Commun., 8 (2006) 102.

[34] T.S. Ahmadi, Z.L. Wang, T.C. Green, A. Henglein, M.A. El-Sayed, Science, 272 (1996) 1924.

[35] J. Solla-Gullón, F.J. Vidal-Iglesias, E. Herrero, J.M. Feliu, A. Aldaz, Electrochem. Commun., 8 (2006) 189.

[36] J. Solla-Gullón, V. Montiel, A. Aldaz, J. Clavilier, J. Electroanal. Chem., 491 (2000) 69.

[37] N. Alexeyeva, K. Tammeveski, A. Lopez-Cudero, J. Solla-Gullon, J.M. Feliu, Electrochim. Acta, 55 (2010) 794. 
[38] Q.S. Chen, S.G. Sun, Z.Y. Zhou, Y.X. Chen, S.B. Deng, Phys. Chem. Chem. Phys., 10 (2008) 3645.

[39] D.A. Cantane, F.H.B. Lima, Electrocatalysis, 3 (2012) 324.

[40] C.A. Angelucci, E. Herrero, J.M. Feliu, J. Phys. Chem. C, 114 (2010) 14154.

[41] E. Herrero, J.M. Feliu, S. Blais, Z. Radovic-Hrapovic, G. Jerkiewicz, Langmuir, 16 (2000) 4779.

[42] E. Herrero, B. Alvarez, J.M. Feliu, S. Blais, Z. Radovic-Hrapovic, G. Jerkiewicz, J. Electroanal. Chem., 567 (2004) 139.

[43] A. López-Cudero, J. Solla-Gullón, E. Herrero, A. Aldaz, J.M. Feliu, J. Electroanal. Chem., 644 (2010) 117.

[44] F. Maillard, E.R. Savinova, P.A. Simonov, V.I. Zaikovskii, U. Stimming, J. Phys. Chem. B, 108 (2004) 17893. 


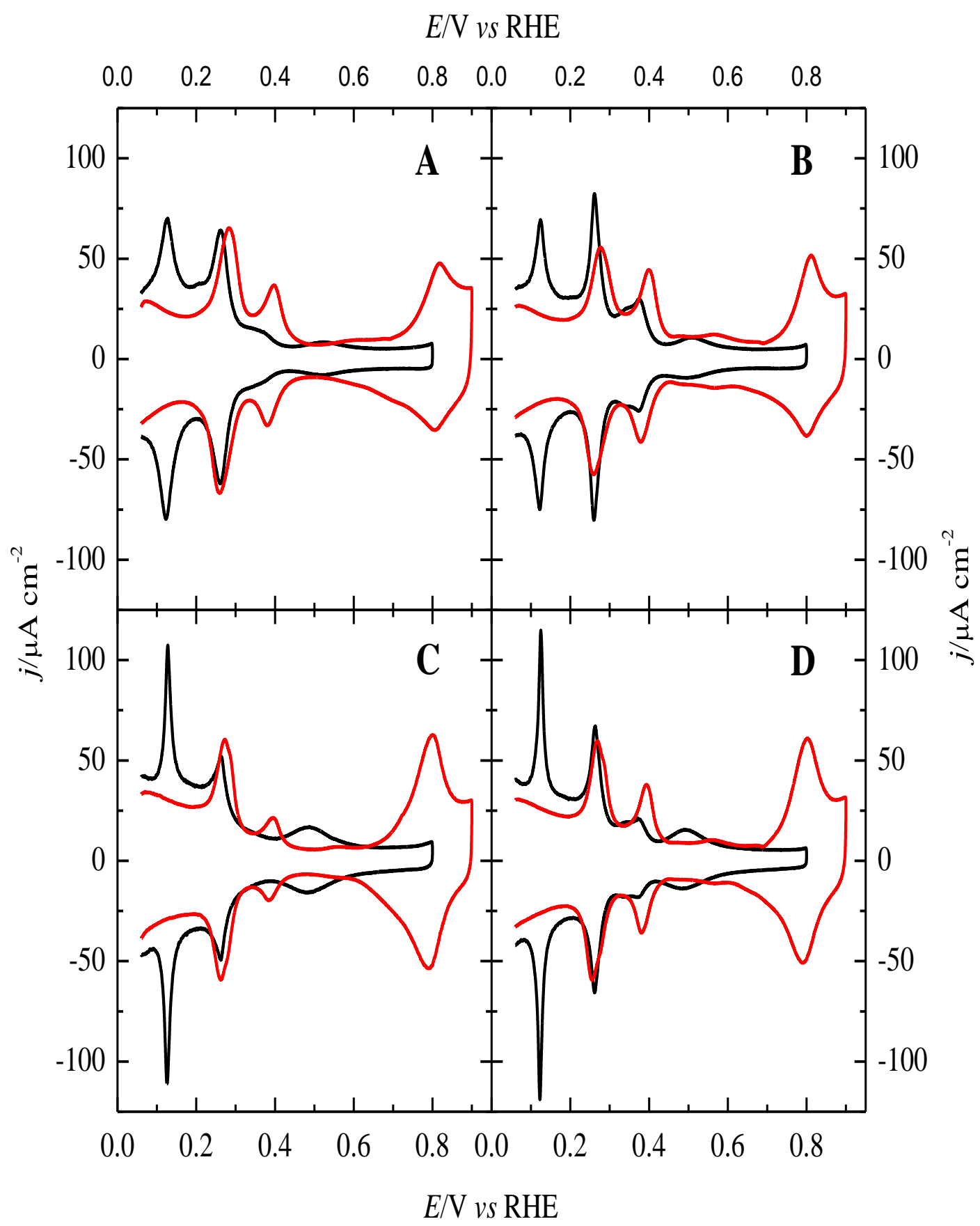

Figure 1. Voltammograms corresponding to: A) $\mathrm{PtNP}_{\text {sphe }}$; B) $\mathrm{PtNP}_{\text {cubic }}$; C) $\mathrm{PtNP}_{\text {tetra }}$, and D) $\mathrm{PtNP}_{\text {trunc }}$ in $0.5 \mathrm{M} \mathrm{H}_{2} \mathrm{SO}_{4}$ (black line) and $0.1 \mathrm{M} \mathrm{NaOH}$ (red line). Scan rate $0.05 \mathrm{~V} \mathrm{~s}^{-1}$. 


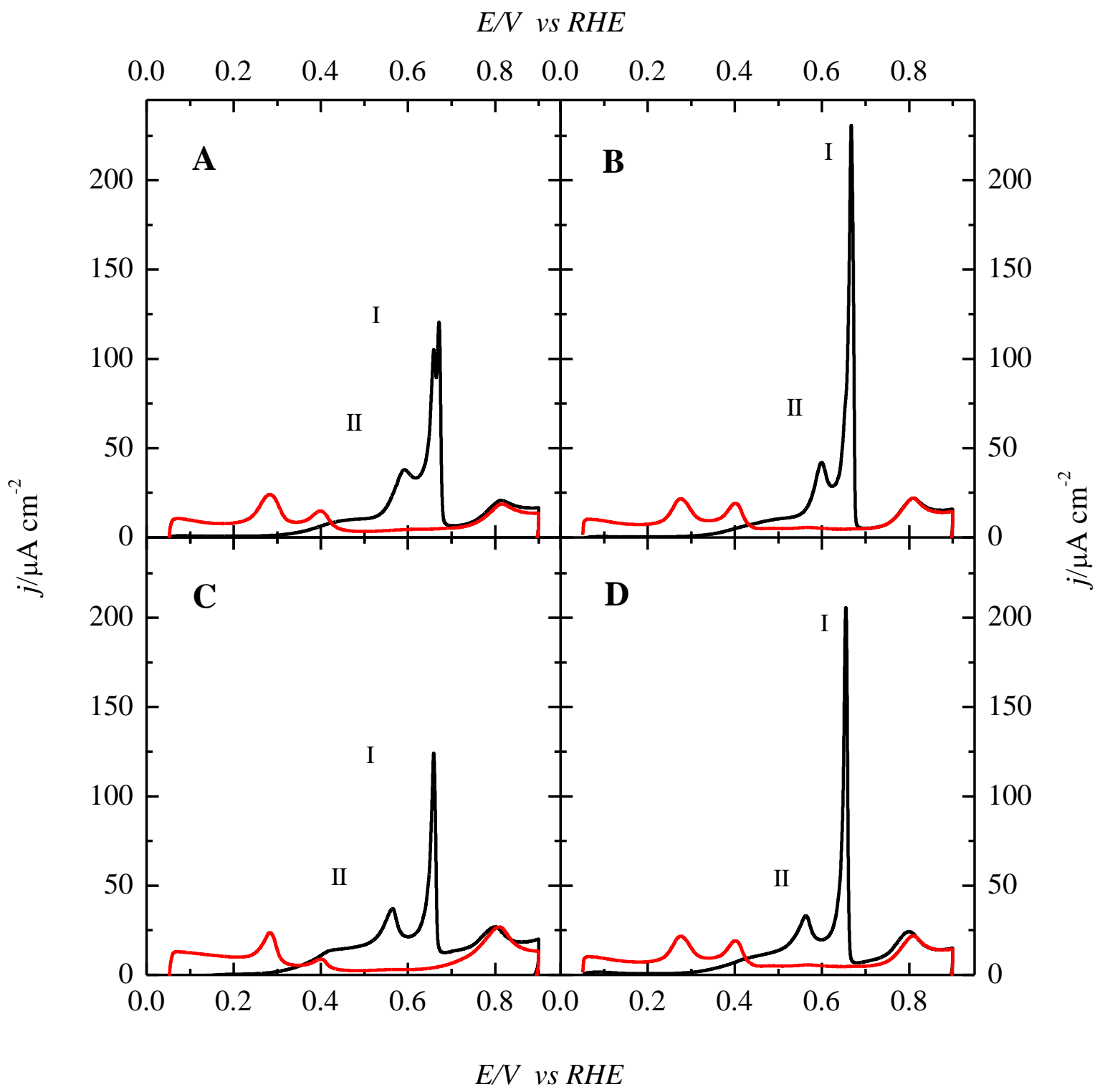

Figure 2. CO stripping (black lines) and blank (red lines) voltammogram in $0.1 \mathrm{M} \mathrm{NaOH}$ for $\mathrm{PtNP}_{\text {sphe }}(\mathrm{A}), \mathrm{PtNP}_{\text {cubic }}(\mathrm{B}), \mathrm{PtNP}_{\text {tetra }}(\mathrm{C})$ and $\mathrm{PtNP}_{\text {trunc }}$ (D). Scan rate: $0.02 \mathrm{~V} \mathrm{~s}^{-1}$. 


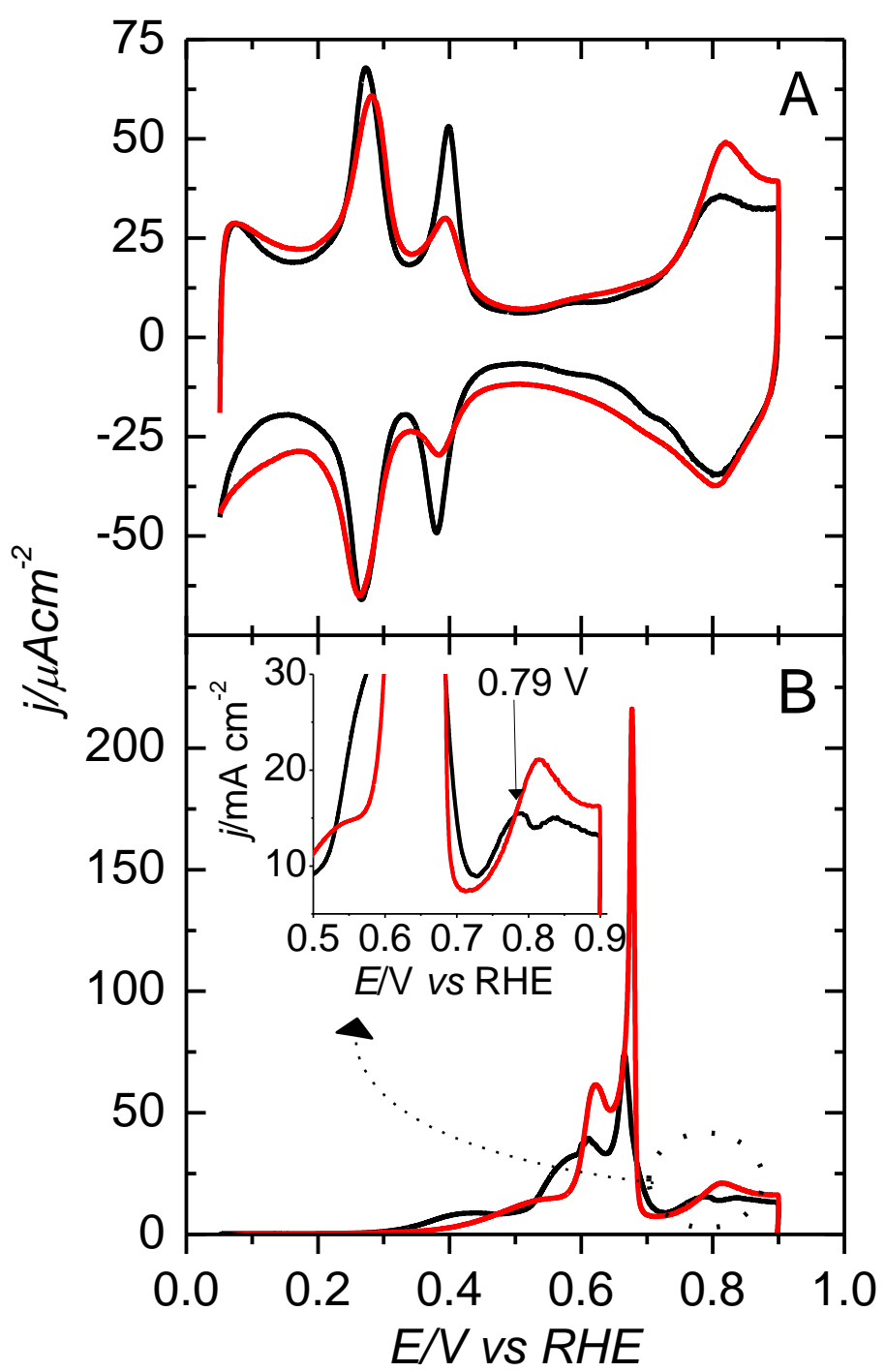

Figure 3. A) Voltammetric profiles of a polycrystalline $\mathrm{Pt}$ electrode (black line) and unsupported spherical Pt nanoparticles $(2-3 \mathrm{~nm}$ ) (red line) in $0.1 \mathrm{M} \mathrm{NaOH}$ (full line). Scan rate: $0.05 \mathrm{~V} \mathrm{~s}^{-1} \mathrm{~B}$ ) $\mathrm{CO}$ stripping voltammograms obtained with a polycrystalline Pt electrode (black line) and with polyoriented Pt nanoparticles $(2-3 \mathrm{~nm}$ ) (red line) in $0.1 \mathrm{M} \mathrm{NaOH}$ (full line) scan rate: $0.02 \mathrm{~V} \mathrm{~s}^{-1}$. 


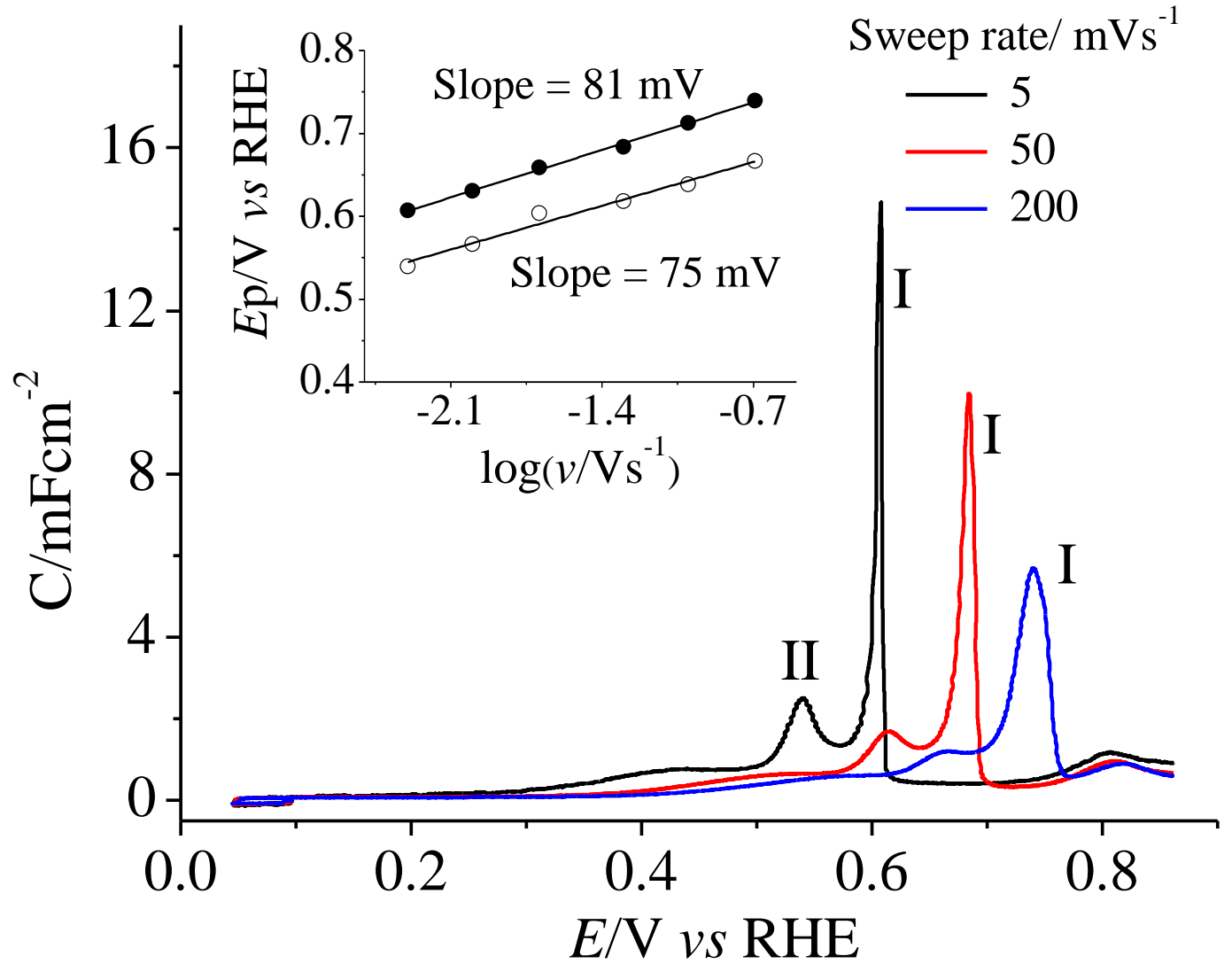

Figure 4. $\mathrm{CO}$ stripping voltammograms at different sweep rate in $0.1 \mathrm{M} \mathrm{NaOH}$ for $\mathrm{PtNP}_{\text {cubic }}$. Insert: Peak potential for $\mathrm{CO}$ electro-oxidation versus the logarithm of the sweep rate. 


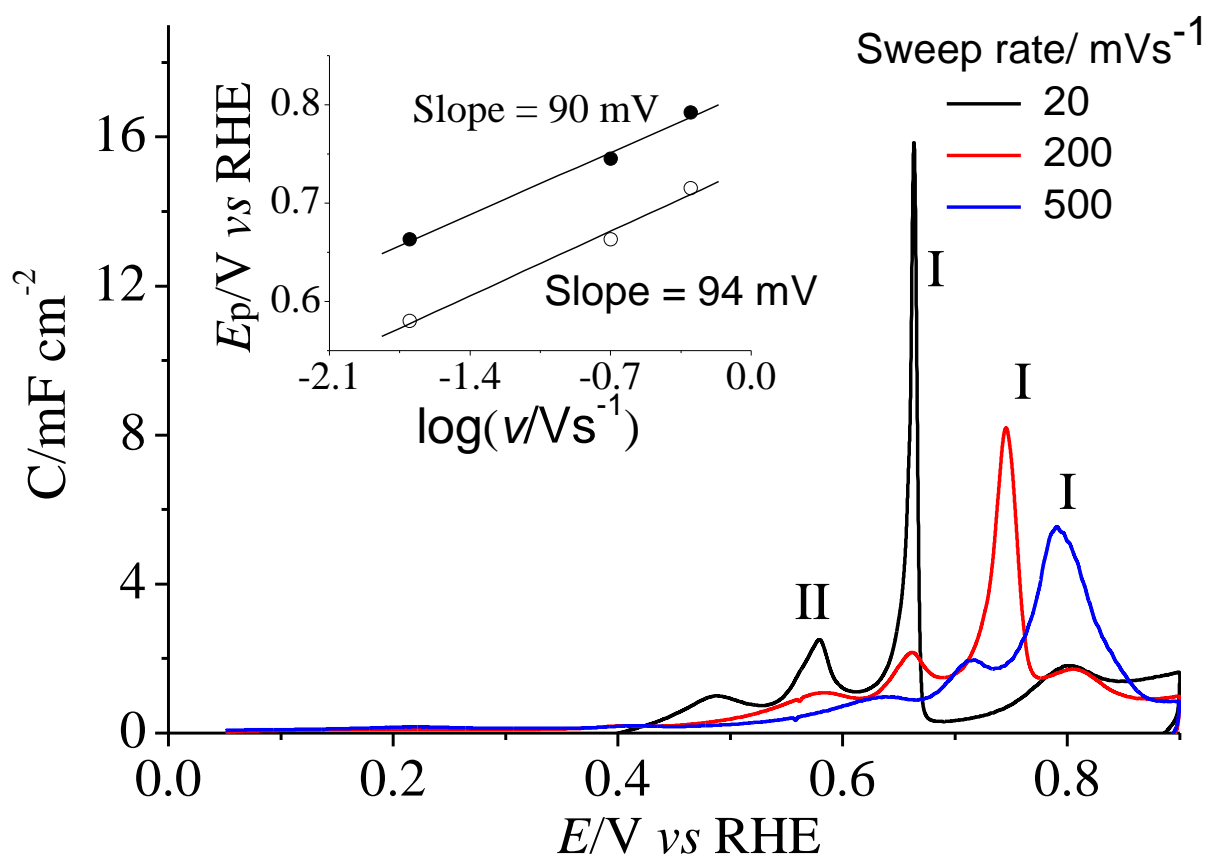

Figure 5. CO stripping voltammograms at different sweep rate in $0.1 \mathrm{M} \mathrm{NaOH}$ for $\mathrm{PtNP}_{\text {trunc. }}$. Insert: Peak potential for $\mathrm{CO}$ electro-oxidation versus the logarithm of the sweep rate. 


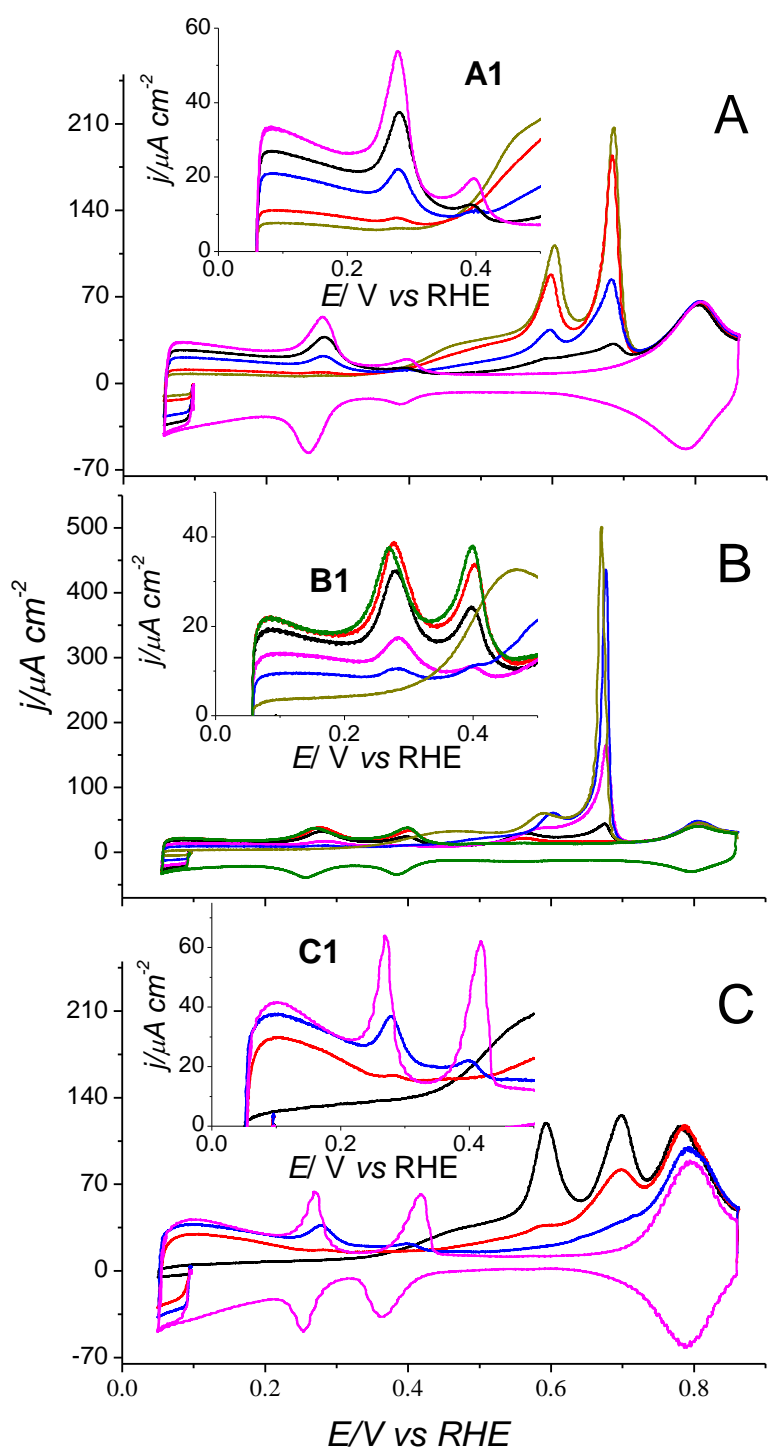

Figure 6. $\mathrm{CO}$ stripping voltammogram at different surface coverage in $0.1 \mathrm{M} \mathrm{NaOH}$ for: A) cubic Pt nanoparticles; B) octahedral Pt nanoparticles; C) and kinked Pt(17 13 11) electrode. Insert: Hydrogen region shown site $\mathrm{CO}$ occupancy. Sweep rate at $0.05 \mathrm{~V} \mathrm{~s}^{-1}$. 


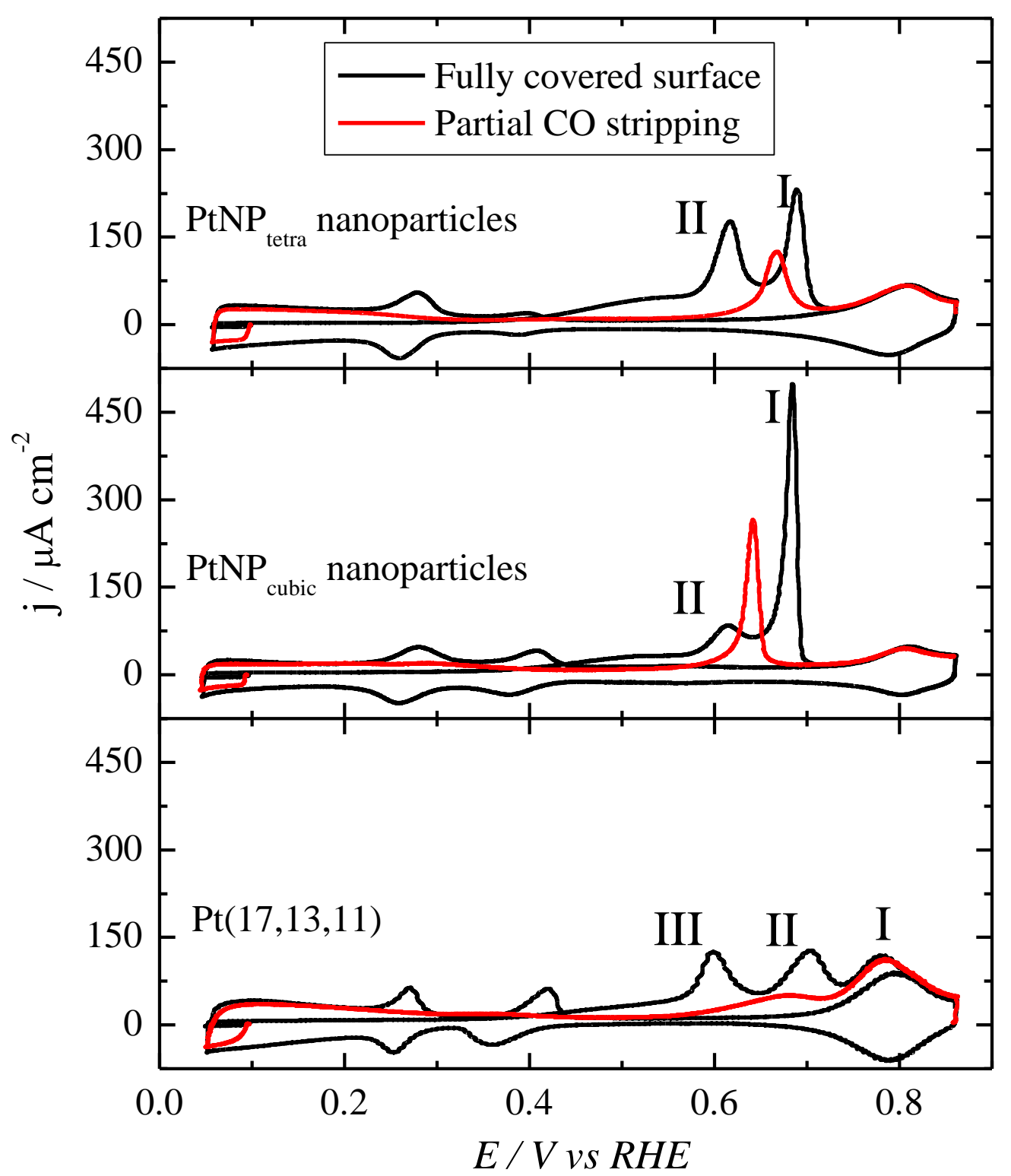

Figure 7. Partial CO oxidation experiments by selecting a low upper potential limit for cubic and octahedral Pt nanoparticles and $\operatorname{Pt}\left(1713\right.$ 11) electrode. Sweep rate at $0.050 \mathrm{Vs}^{-1}$. 


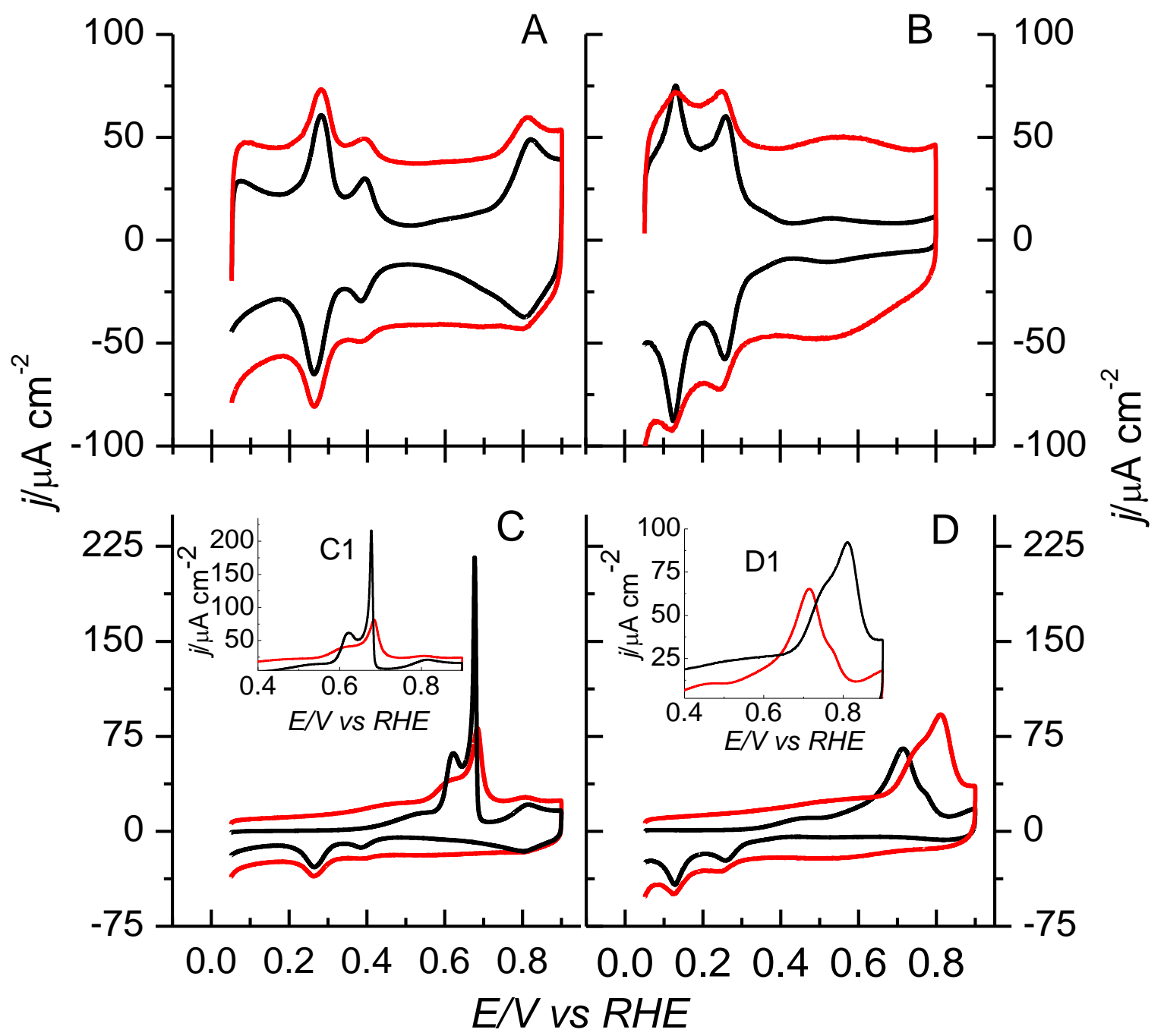

Figure 8. Voltammetric profiles of $\mathrm{Pt} / \mathrm{C}(10 \%)$ Vulcan (red line) and unsupported $\mathrm{Pt}$ (black line) samples at $0.05 \mathrm{~V} \mathrm{~s}^{-1}$ : A) in $0.1 \mathrm{M} \mathrm{NaOH}$; B) in $0.5 \mathrm{M} \mathrm{H}_{2} \mathrm{SO}_{4}$. CO stripping voltammograms obtained with $\mathrm{Pt} / \mathrm{C}(10 \%)$ Vulcan (black line) and unsupported $\mathrm{Pt}$ (red line) samples at $0.02 \mathrm{~V}$ $\mathrm{s}^{-1}: \mathrm{C}$ ) in $0.1 \mathrm{M} \mathrm{NaOH}$; D) in $0.5 \mathrm{M} \mathrm{H}_{2} \mathrm{SO}_{4}$. 
